\title{
Characterization of Plasmonic Effects in AuNP+rGO Composite as a Sensing Layer for a Low-cost Lab-on-chip Biosensor
}

\author{
Alessandro Fantoni ${ }^{1,2}$, Vladan Stojkovic ${ }^{1}$, Miguel Fernandes ${ }^{1,2}$, Paula Louro $^{1,2}$, \\ Manuela Vieira $^{1,2,5}$, Elisabete C. B. A. Alegria ${ }^{3,4}$, Ana P. C. Ribeiro ${ }^{4}$, \\ Ana Carvalho ${ }^{3}$, and M. Gabriela Almeida ${ }^{6,7}$ \\ ${ }^{1}$ ADEETC-ISEL-Instituto Politécnico de Lisboa, Lisbon 1949-014, Portugal \\ ${ }^{2}$ CTS-UNINOVA, Caparica, Portugal \\ ${ }^{3}$ ADEQ-ISEL-Instituto Politécnico de Lisboa, Lisbon 1949-014, Portugal \\ ${ }^{4}$ CQE-Instituto Superior Técnico, Universidade de Lisboa, Lisbon 1049-001, Portugal \\ ${ }^{5}$ DEE-FCT-UNL, Caparica, Portugal \\ ${ }^{6}$ UCIBIO-REQUIMTE, Caparica, Portugal \\ ${ }^{7}$ CiiEM, Instituto Universitário Egas Moniz, Caparica, Portugal
}

\begin{abstract}
This work deals with the production of a low-cost disposable biosensor for point of care applications. The proposed sensor is a plasmonic structure based on the Localized Surface Plasmon Resonance (LSPR) interaction of metal nanoparticles (MNPs), embedded into a matrix of reduced Graphene Oxide (rGO). After proper functionalization with selective antibodies $(\mathrm{Ab})$, the efficiency of light extinction is controlled by slight changes of the refractive coefficient induced by the concentration of biomarkers trapped by the antibodies on the sensor surface. This work reports a study about the applicability of rGO as a support for gold nanoparticles (AuNPs) for preparing the functionalized LSPR sensing layer. AuNPs are prepared with an economic and eco-friendly method using phytochemicals present in tea extract at room temperature, while a modified Hummer's method is used to synthesize rGO. The resulting AuNPs-rGO composites are studied in terms of UV-VIS spectroscopy spectral light transmission and plasmonic resonance. The overall analysis is supported by simulation results, obtained by Mie analysis, about the LSPR effect in AuNPs-rGO and its dependence on the biomarker concentration.
\end{abstract}

\section{INTRODUCTION}

Graphene has attracted great attention in the scientific community because of its combination of optical and mechanical interesting properties, opening opportunities for applications over the entire light spectrum, ranging from the UV to the infrared boosting its inclusion in in a large variety of biosensing applications [1]. Reduced Graphene Oxide (rGO) is a kind of graphene derived by chemical processes. rGO possesses equivalent optical properties but its production is easier and cheaper. rGO) has been recently proposed and studied as a nanocomposite in conjunction with gold nanoparticles [2] and proposed as a biosensing element for aflatoxins in a practical device [3]. The chemical composition of rGO supports the functionalization with specific antibodies or enzymes, allowing an improvement of the biocompatibility. From this point of view, a functionalized surface based on rGO drives a cost reduction of the sensor realization. In this work, rGO is studied in conjunction with the local surface plasmonic resonance (LSPR) produced by gold nanospheres (AuNPs). Results are presented about light transmission and absorption of rGO decorated with AuNPs, evaluating the possibility of using this material as the sensing element for a protein sensor device.

The proposed device structure is depicted in Figure 1. The active sensing layer is a composite of gold nanoparticles, embedded in a matrix of rGO (AnNPS+rGO). The light transmitted across this layer is governed by the Localized Surface Plasmon Resonance (LSPR) interaction of Au nanoparticles. Such an interaction depends strongly on the optical properties (i.e., the refractive index) of the medium surrounding the nanoparticles. The useful LSPR interacting wavelength spectrum of AuNPs is located in the 500-700 nm range, which is the part of the spectrum where we find the optimal spectral responsivity of PECVD thin film photodiodes, like amorphous or nanocrystalline silicon devices [4]. This condition allows the realization of an integrated device whose optoelectronic readout is performed by an amorphous silicon photodiode that by measuring the spectral photocurrent is able to monitor the intensity of the LSPR effect and the light transmission properties of the AuNPS+rGO layer. Following the functionalization functionalization of the AuNPS+rGO layer with selective antibodies, (and consequently the light transmitted and measured by the underlying photodiode) is controlled by the slight changes of the refractive index induced by the biomarker 
concentration, as captured by the surface antibodies. The goal of the work hereby presented is the study of the plasmonic properties of the AuNPs-rGO composites for different light wavelength and nanosphere dimension. The focus of this study is on the LSPR absorption profile, measured by $\mathrm{UV}-\mathrm{Vis}$ spectroscopic analysis. A theoretical analysis is also presented, based on the Mie theory, about the LSPR of AuNPs with different dimension, diluted in water and in composition with rGO.

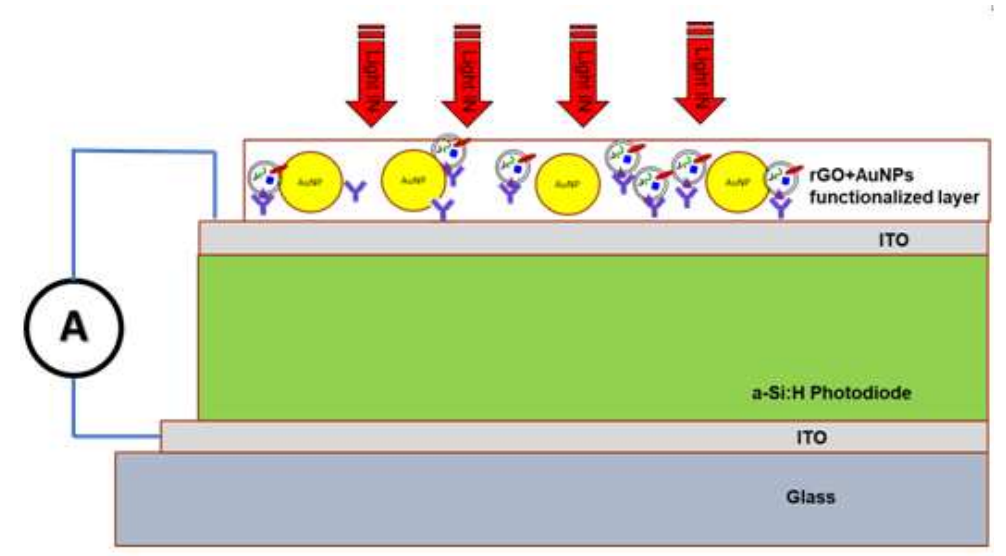

Figure 1: Schematics of the LSPR sensor based on rGO+AuNPS light transmission properties.

\section{SIMULATION: MIE ANALYSIS}

\subsection{Theory}

The most effective theoretical approach to the characterization of the LSPR effect produced by metal nanoparticles is described in the work of Gustave Mie [5], realized in the begging of the XX century as a straightforward solution of the Maxwell equations in spherical coordinates, with symmetrical boundary conditions. More recently the Mie theory has been retroposed in a manner suitable for a direct implementation in a computer program [6]. The Mie approach is limited to the spherical geometry of the nanoparticles and describes their interaction with the incident light in terms of the light extinction efficiency $\left(Q_{\text {ext }}\right)$, as the sum of absorption and scattering phenomena $\left(Q_{a b s}\right.$ and $\left.Q_{s c a}\right)$. Using the same approach, the Mie Theory can be also applied to the case of core shell spherical naparticles.

Given a metal, the plasmonic properties of nanoparticles are determined, by their shape and size. According to the Mie theory the Local Plasmonic Resonance is dependent on the interaction between the nanoparticle and the surrounding medium. A simplified expression for the particle (complex) polarizability for a simple spherical geometry is quantified as:

$$
\alpha=4 \pi r^{3} \frac{\varepsilon_{m}-\varepsilon_{s}}{\varepsilon_{m}+2 \varepsilon_{s}}
$$

where $r$ is the radius of the nanosphere, $\varepsilon_{m}$ and $\varepsilon_{s}$ are the permittivity of the metal and of the surrounding medium, respectively. The optical properties for metals and dielectrics, necessary for our simulations as required by the Mie theory, have been taken by literature [7-10].

\subsubsection{Simulation Results}

A Direct Mie analysis has been used to simulate the LSPR of gold nanospheres embedded in Graphene, rGO and, for comparative purpose, in a colloidal solution [11]. As expected, our results show that the wavelength for the LSPR peak depends strongly on the nanoparticle radius. It is also possible to detect an increasing multipolar response and a peak broadening for large sphere radius. On the other hand, a sharp and localized peak can be observed when the NPs radius is small. When the size of the NPs is as small as $20 \mathrm{~nm}$, absorption is the dominant phenomena and the most part of light extinction for these cases is due to the enhance absorption caused by the LSPR. The simulated light extinction for $\mathrm{Au}$ nanospheres with a radius in the range 10-100 nm embedded in Graphene, rGO and distilled water is depicted in Figure 2. The resonance peaks wavelength and width vary greatly with the surrounding medium. For rGO the peak is located in the red part of the spectrum, in the 600-700 range, depending on the NP size. This result is of 
greatly importance for our projected applications, as the spectral sensitivity of a-Si:H is still good in this range, becoming much lower when entering in the infrared region (wavelengths above $700 \mathrm{~nm}$ ). The extinction efficiency of a $40 \mathrm{~nm}$ nanosphere covered by a thin layer of rGO with increasing thickness is presented in Figure 3. The rGO cladding layer produces the effect of red-shifting the LSPR peak, proportionally to the cladding thickness. This behaviour is confirmed in the result of Figure 4, where can be observed that that according with the Mie Theory, the central wavelength of LSRP peak depends directly on the rGO concentration.
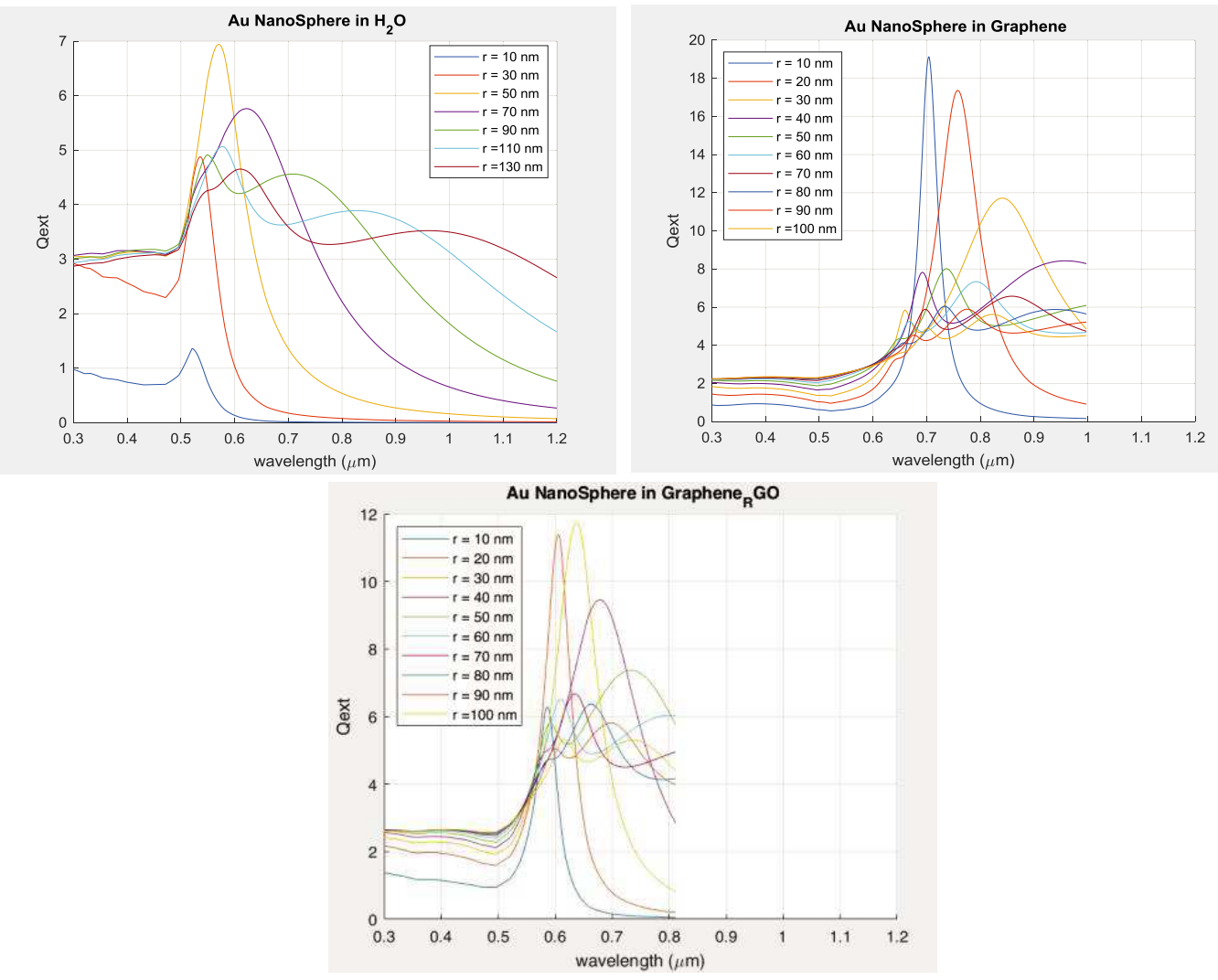

Figure 2: Extinction efficiency, calculated by Mie analysis, for gold nanospheres with radius in the $10-100 \mathrm{~nm}$ range, in diluted water, graphene and reduced graphene oxide (rGO).

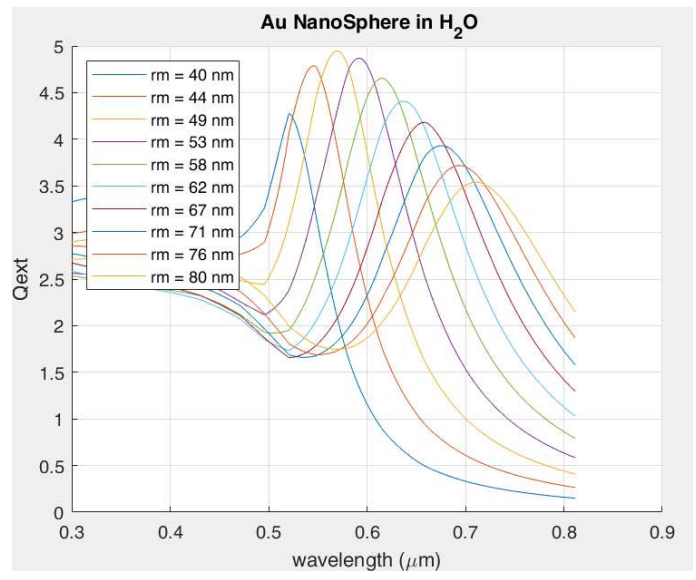

Figure 3: Extinction efficiency, calculated by Mie analysis, for gold nanospheres with $40 \mathrm{~nm}$ radius covered by a rGO cladding layer with thickness between 0 and $40 \mathrm{~nm}$ diluted in water. 

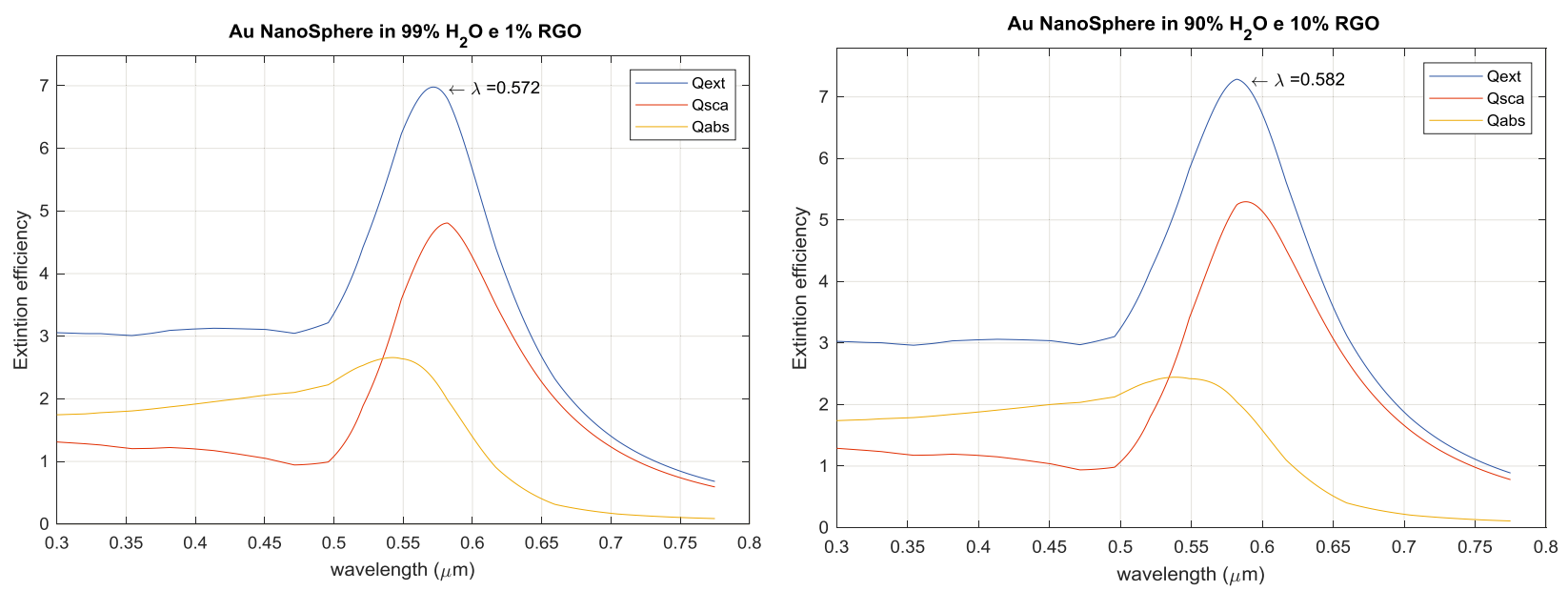

Figure 4: Extinction efficiency, calculated by Mie analysis, for gold nanospheres with $50 \mathrm{~nm}$ radius diluted in a mixture of water and rGO with different concentrations.

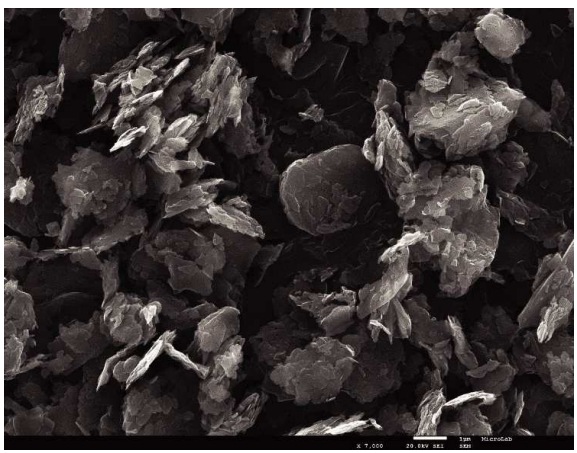

Figure 5: SEM image of the rGO used in the experimental measurements.

\section{EXPERIMENTAL}

\subsection{A Preparation of $\mathrm{rGO}$}

The synthesis of graphene oxide (GO) was performed in accord to a reported method [12] in which the graphite $(1.0 \mathrm{~g})$ is added to a solution of $\mathrm{NaNO}_{3}(0.5 \mathrm{~g})$ in concentrated $\mathrm{H}_{2} \mathrm{SO}_{4}(30 \mathrm{~mL})$. The mixture was cooled to $0^{\circ} \mathrm{C}$ and $\mathrm{KMnO}_{4}(3.0 \mathrm{~g})$ was added and the temperature kept below $20^{\circ} \mathrm{C}$. The mixture was stirred for 1 hour at $35^{\circ} \mathrm{C}$, and then the temperature was increased to $85^{\circ} \mathrm{C}$ and the solution diluted with deionized water $(46 \mathrm{~mL})$. The mixture was heated to $100^{\circ} \mathrm{C}$ and maintained at this temperature for $30 \mathrm{~min}$. Warm deionized water $(140 \mathrm{~mL})$ is added to the mixture, followed by the dropwise addition of a $50 \%$ aqueous solution of $\mathrm{H}_{2} \mathrm{O}_{2}(15 \mathrm{~mL})$, and the solution is stirred for 30 min. After this period the mixture is centrifuged and then washed with $5 \%$ aqueous $\mathrm{HCl}$ solution $(1 \mathrm{~L})$ to remove the metal ions, and then with deionized water $(1 \mathrm{~L})$ to remove the excess of acid. GO is finally obtained after keeping the sample to dry for 12 hours at $60^{\circ} \mathrm{C}$. Reduced graphene oxide (rGO) is synthesized through a solvothermal method. In a typical synthesis the GO is dispersed in ethylene glycol $(60 \mathrm{~mL})$ and sonicated for $1 \mathrm{~h}$, followed by the subsequent addition of aqueous $\mathrm{NH}_{3}(1.6 \mathrm{~mL})$, and stirred for $1 \mathrm{~h}$. The rGO is mixed with AuNPs through a mechanical process. Figure 5 shows SEM (Scanning Electron Microscopy) image of the synthesized rGO.

\subsection{Preparation of rGO-AuNPs Composite}

Commercially available gold spherical nanoparticles with radius in the $20-50 \mathrm{~nm}$ range were used [13] to prepare the rGO-AuNPs composite. The prepared AuNPs are uniform in size and shape, being their LSPR behaviour in accordance with that described by the Mie analysis applied to the ideal case of $\mathrm{Au}$ spheres diluted in water [14]. For a uniform dispersion, AuNPs and rGO were mixed with an ultrasonic bath at $44 \mathrm{kHz}$ for 15 minutes, and after that with a sonicator at $20 \mathrm{kHz}$, for 1 minute. The rGO concentration in the resulting colloidal solutions $(3 \mathrm{~mL})$ are in the 0.5 to $4 \mathrm{mg}$ range for solutions containing $5.8 \times 10^{-5} \mathrm{~g} / \mathrm{mL}$ of gold.

AuNPs have also been prepared through an economic and eco-friendly method, at room tem- 
perature, using a metallic salt $\left(\mathrm{HAuCl}_{4}\right)$ and phytochemicals present in tea extract as both the reducing and capping agent. This green method for AuNPs synthesis targets the production of a low cost and disposable sensor device. Transmission electron microscopy (TEM), Scanning electron microscopy (SEM) and energy dispersive X-ray spectroscopy (EDX) and UV-Vis spectroscopy were used to characterize the AuNPs (Figure 6). The EDX plot shows the presence of gold, together with other elements derived from the tea extracts. The concentration of gold and polyphenols can be in certain manner controlled during the process, but the presence of other elements must be considered. The SEM images show that the polyphenols form large aggregates allowing the controlled growing of AuNPs on their surface. TEM image analysis allows to deduce that in most cases the AuNPs are covered by a layer of polyphenols. TEM image it is possible to deduce that in the most of cases the AuNPs are capped by a layer of polyphenols. The nanoparticles thus produced are mostly spherical and although the size is not so uniform it is in the $10-20 \mathrm{~nm}$ range.

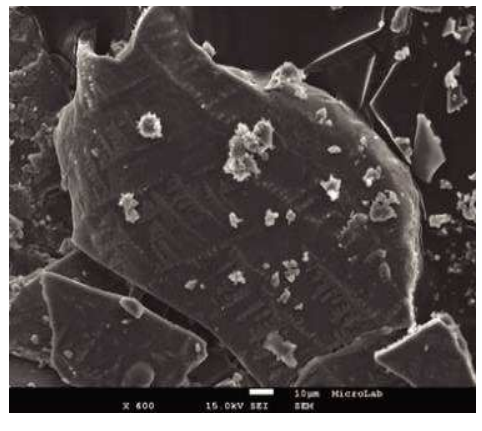

(a)

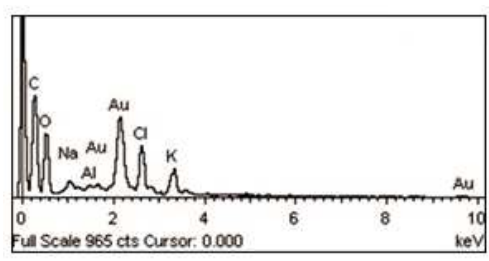

(c)

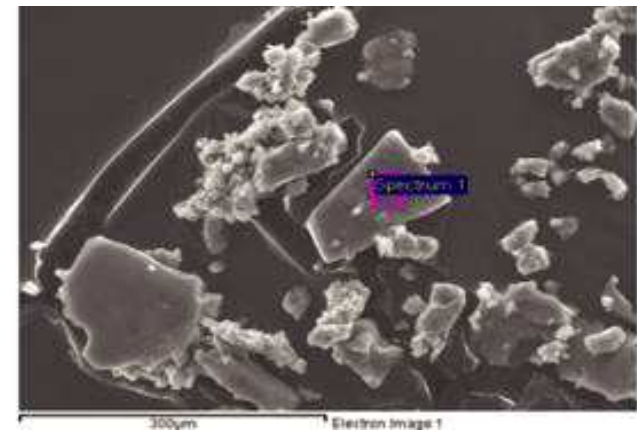

(b)

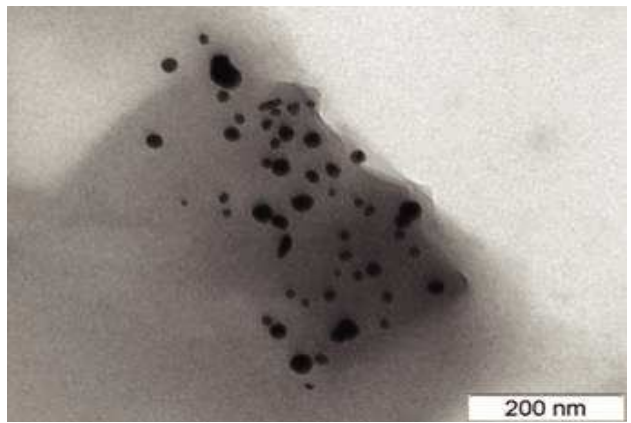

(d)

Figure 6: Characterization of the AuNPs produced by using phytochemicals present in tea extract, at room temperature. SEM image of (a) $5 \%$ tea solution and (b) tea with AuNPs. (c) EDX graph of $5 \%$ tea solution and AuNPs. (d) TEM image of AuNPs.

\subsection{UV-VIS Measurements}

In the characterization of commercial AuNPs although, no modification in the LSPR wavelength was detected for nanoparticles with radius less than $40 \mathrm{~nm}$, the influence of the rGO coupling to AuNPs could be clearly observed for $50 \mathrm{~nm}$ radius nanoparticles. Figure 7 shows the measured light absorption profile in the visible range, for different concentrations of rGO. The presence of rGO results in a general reduction of light transmission over the entire spectrum, in comparison to the solutions containing exclusively AuNPs. The LSPR peak, to be ascribed to the plasmonic behaviour of the nanospheres is well defined in all the measured cases. The central wavelength for this plasmonic resonance is red shifted by the AuNPs-rGO coupling, as reported in Figures 7 and 8, where the peak wavelength is plotted as a function of the rGO concentration. This is in accordance with the simulation results described in the previous section. Comparing the simulation and the experimental results we can conclude that even if there is no complete covering of the AuNPs by the rGO (that is the cladding has by no means a uniform thickness, as simplistically assumed in the Mie analysis) the plasmonic resonance wavelength is affected by the difference in the refractive index introduced by the rGO and the shift of the LSPR wavelength, experimentally observed, can be interpreted as a measure of the interaction between the AuNPs and the presence of rGO within the surrounding medium. If no rGO is introduced, the observed LSPR is almost in perfect agreement with the simulated data. The red shifting caused by the increasing rGO concentration 


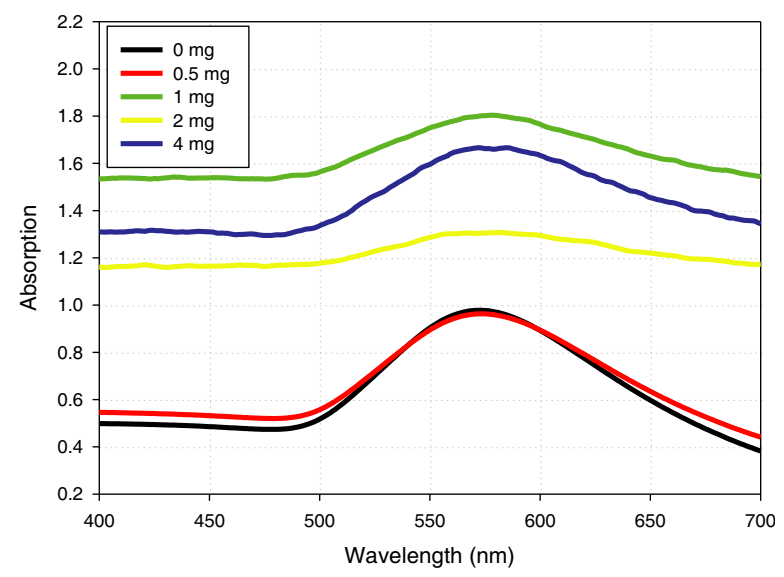

Figure 7: Measured absorption profile of the rGO+AuNPs colloidal solution with different concentration of rGO.

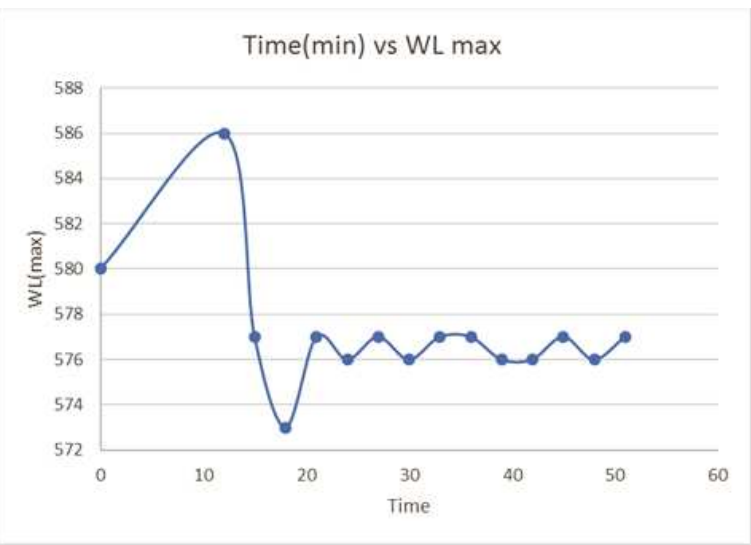

Figure 9: Time analysis of the LSPR wavelength.

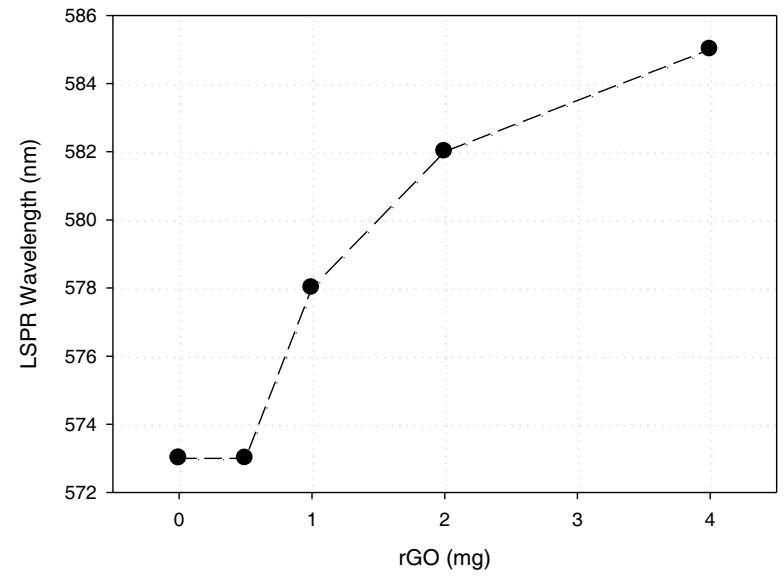

Figure 8: LSPR wavelength measured by UV-VIS absorption of rGO+AuNPs colloidal solution, as a function of the rGO concentration. Nanoparticle radius is $50 \mathrm{~nm}$.

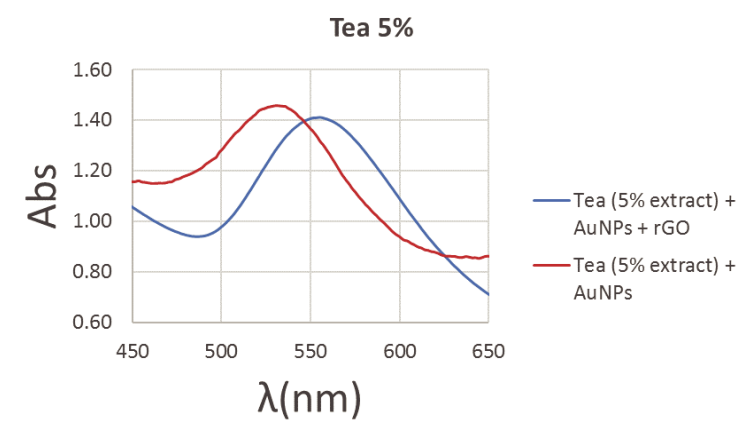

Figure 10: UV-VIS absorption spectra. LSPR peak of AuNPs obtained with $5 \%$ tea extract, with and without reduced Graphene Oxide (rGO).

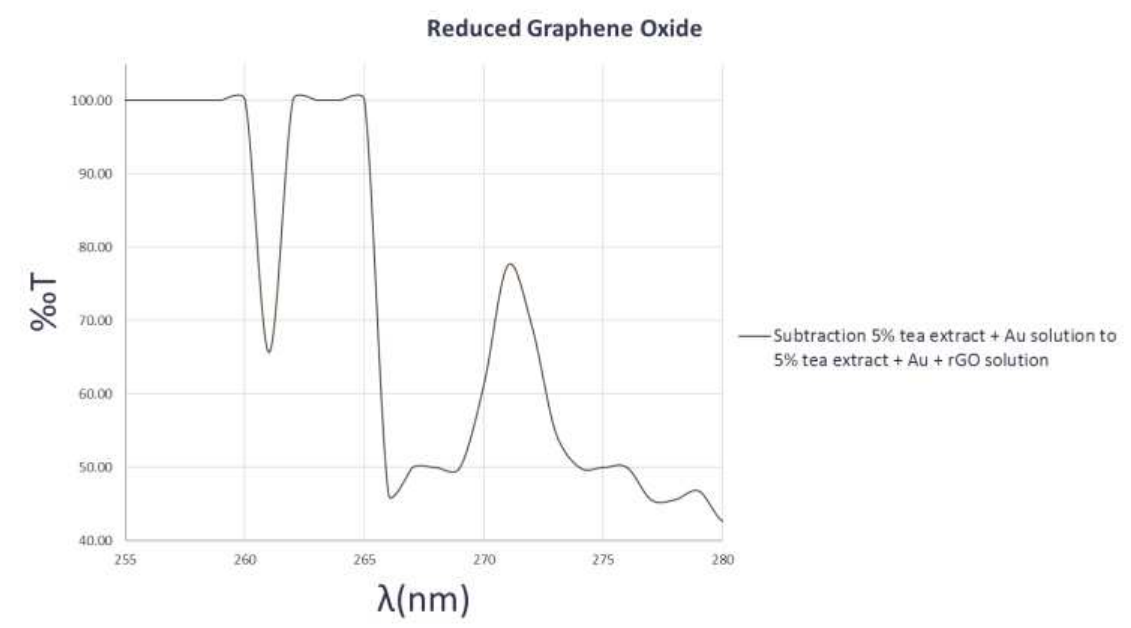

Figure 11: UV-VIS. Characteristic band of Reduced Graphene Oxide.

can be ascribed to a better and more complete cladding of the nanoparticles. It should be noted that the resonance peak remains unique and we cannot observe superposition of two separate peaks related to the cladded or not-cladded NPs. The LSPR shift remains stable up to 50 minutes after the ultrasonic mixing, as reported in Figure 9. In Figure 10 is reported the absorption profile, measured by UV-VIS, of the AuNPs produced through a quite simple and environmentally benign 
process. The LSPR peak is well defined, and the central wavelength of the resonance is $540 \mathrm{~nm}$, corresponding to the value provided by the Mie theory for nanoparticles of small diameter. The AuNPs-rGO composite shows a remarkable shift of about $25 \mathrm{~nm}$. This result is valuable and promising, as it demonstrates that the nanoparticles produced by the green method, even if size is not uniform and if they are capped by tea phenolic residuals, maintain the full potential for LSPR sensor applications. The effective presence of rGO composite is confirmed by the absorption measurement in the UV range, reported in Figure 11.

\section{CONCLUSION}

Considering that the AuNPs-rGO composite is a promising candidate as a support for antibodies functionalization as the sensing layer if a photonic biosensor, we have demonstrated that it is possible to obtain a uniform mixture of AuNPs and rGO using mechanical processes such as ultrasonic bath or ultrasonic sonication. Anyway, when the AuNP diameter is smaller than $100 \mathrm{~nm}$ no modification of the LSPR response can be observed. For $100 \mathrm{~nm}$ AuNPs the mixture with rGO results in a red shift of the LSPR which depends on the rGO concentration. Such red-shift in the AuNPs+rGo composites has been observed using commercial high quality AuNPs and in locally produced using a low-cost process. On the way for a biosensor application, future work will focus on an efficient method for functionalization with antibodies and the deposition of the composite on top of a photodiode photo-active surface.

\section{ACKNOWLEDGMENT}

Research supported by EU funds through the FEDER European Regional Development Fund and by Portuguese national funds by FCT -Fundação para a Ciência e a Tecnologia with projects PTDC/NAN-OPT/31311/2017, SFRH/BPD/102217/2014 and by IPL IDI\&CA/2018/aSiPhoto.

\section{REFERENCES}

1. Pumera, M., "Graphene in biosensing," Materials Today, Vol. 14, Nos. 7-8, 308-315, 2011.

2. Osváth, Z., A. Deák, K. Kertész, G. Molnár, G. Vértesy, D. Zámbó, and L. P. Biró, "The structure and properties of graphene on gold nanoparticles," Nanoscale, Vol. 7, No. 12, 55035509, 2015.

3. Guo, W., L. Wu, K. Fan, D. Nie, W. He, J. Yang, and Z. Han, "Reduced graphene Oxide-gold nanoparticle nanoframework as a highly selective separation material for aflatoxins," Scientific Reports, Vol. 7, No. 1, 14484, 2017.

4. Vieira, M., S. Koynov, A. Fantoni, and R. Schwarz, "Wide spectral response in $\mu c-S i$ : H photodiodes," Thin Solid Films, Vol. 296, Nos. 1-2, 164-167, 1997.

5. Mie, G., "Beiträge zur optik trüber medien, speziell kolloidaler metallösungen," Annalen der Physic, Vol. 330, No. 3, 377-445, 1908.

6. Bohren, C. F. and D. R. Huffman, Absorption and Scattering of Light by Small Particles, John Wiley \& Sons, 2008.

7. Palik, E. D., Handbook of Optical Constants of Solids, Vol. 3, Academic press, 1998.

8. Olmon, R. L., B. Slovick, T. W. Johnson, D. Shelton, S. H. Oh, G. D. Boreman, and M. B. Raschke, "Optical dielectric function of gold," Physical Review B, Vol. 86, No. 23, 235147, 2012.

9. McPeak, K. M., S. V. Jayanti, S. J. P. Kress, S. Meyer, S. Iotti, A. Rossinelli, and D. J. Norris, "Plasmonic films can easily be better: Rules and recipes," ACS Photonics, Vol. 2, 326-333, 2015.

10. Schmiedova, V., et al., "Physical properties investigation of reduced graphene oxide thin films prepared by material inkjet printing," Journal of Nanomaterials, 2017.

11. Fantoni, A., M. Fernandes, Y. Vygranenko, P. Louro, M. Vieira, E. C. Alegria, et al., "A simulation study of surface plasmons in metallic nanoparticles: Dependence on the properties of an embedding a-Si: H matrix," Physica Status Solidi (a), Vol. 215, No. 3, 1700487, 2018.

12. Chen, J., B. Yao, C. Li, and G. Shi, "An improved Hummers method for eco-friendly synthesis of graphene oxide," Carbon, Vol. 64, 225-229, 2013.

13. BBI Solutions, 73 Ty Glas Avenue Cardiff, UK, https://www.bbisolutions.com.

14. Fantoni, A., M. Fernandes, Y. Vygranenko, P. Louro, M. Vieira, R. P. O. Silva, and E. C. Alegria, "Analysis of metallic nanoparticles embedded in thin film semiconductors for optoelectronic applications," Optical and Quantum Electronics, Vol. 50, No. 6, 246, 2018. 